\title{
Designing of Computer Aided Diagnostic System for the Identification of Exudates in Retinal Fundus Images
}

\author{
${ }^{1}$ Santosh Kumar Mishra, ${ }^{2}$ Deepti Mittal and ${ }^{3}$ Ramesh Kumar Sunkaria \\ ${ }^{1,2}$ Electrical and Instrumentation Engineering Department, Thapar University, Patiala (Punjab), India; \\ ${ }^{3}$ Department of electronic and communication, Dr.B.R. Ambedkar NIT Jalandher, jalandher, India \\ ${ }^{1}$ Santosh.mishra80135@gmail.com; 2deepti.mittal@thapar.edu; ${ }^{3}$ sunkariark@gmail.com
}

\begin{abstract}
Macular edema is an advance stage of diabetic retinopathy which affects central vision of diabetes patients. The main cause of edema is the appearance of exudates near or on macular region in human retina. If the exudates are present in the macular region of retina, it will lead to diabetic macular edema. Early detection of macular edema in diabetic patients paves a path for prevention from blindness. The automatic system for early detection of diabetic macular edema should identify all possible exudates present on the surface of retina. In the proposed work, a computer added diagnosis system is design for the identification of the exudates in color retinal fundus images. The system consists of three stages; candidate exudates detection, feature extraction and classification. The system is designed with (i) background estimation, morphological reconstruction, normalization for candidate exudates detection (ii) Gray level co-occurrence matrix for feature extraction and (iii) support vector machine for classification. The classifier classifies in between the region of exudates and non-exudates. The system performance is evaluated in terms of the parameters such as sensitivity, specificity, mathews correlation coefficient, positive predicative value, and accuracy whose values are $88.23 \%, 100 \%, 88.23 \%, 100 \%, 93.75 \%$ respectively.
\end{abstract}

Keywords: Macular edema, Diabetic retinopathy, Exudate, Retina fundus images, Morphological reconstruction, Normalization, Candidate exudates detection,

\section{Introduction}

Diabetic retinopathy (DR) is a progressive eye disease that currently affects millions of people worldwide. Diabetic macular edema (DME) is a complication of diabetic retinopathy and it is a common cause of vision impairment and blindness [1]. DME is one of the complications caused by diabetes; DME may lead to visual damages in people at a working age. However, the risk of vision loss and blindness may be reduced if DME is detected early, and followed by appropriate treatment. DME occurs from swelling of the retina in diabetic patients due to leaking of fluid from micro aneurysms within the macula. This presentence of fluid causes retina thickening in diabetic patients which is termed lipid deposits also known as exudates. Exudates appear as bright structures with well-defined edges and variable shapes.

Study in medical imaging has shown that most of the exudates screen themselves in the human retina. This leads to a clear road for researchers to device and improve the method for analyzing exudates. 
Therefore, specialized image processing method is used to obtain proper identification of exudates in retinal fundus images [2-3]. These image processing methods may help to medical professionals in timely diagnosis of DME and improve medical facilitation available to the patients.

There are two types of macular edema, (i) Non Clinically Significant Macular Edema (Non-CSME) and (ii) Clinically Significant Macular Edema (CSME). Non-CSME is a mild form of edema in which there are no symptoms of the disease because the locations of exudates are at a distance from fovea and the central vision is not affected. CSME is the severe form of edema in which the exudates leak out and get deposited very close to or on fovea affecting central vision of the eye [4]. Fig.1. is an example of retinal fundus image showing digital image of human retina along with its main components and exudates.

Many methods for diagnosing macular edema and other retinal diseases are discussed in literature [5-7]. Also exudates segmentation and classification method presented in the literature are based on: Thresholding and morphological methods, thresholding method analysis [8], Thresholding methods identify the exudates by local and global analysis of threshold whereas morphological methods first identify all the structure of predictable shape and removed them afterwards for easy detection of exudates in retinal fundus images [9-12]. Also, there are number of classification method to classify various lesion types in retinal images such as drusen, cottonwood spot, exudates [13-14]. In this work classification is performed in between exudates and non-exudates region on pixel to pixel basis, which will further aid to ophthalmologist in diagnosis of patient diabetic macular edema.

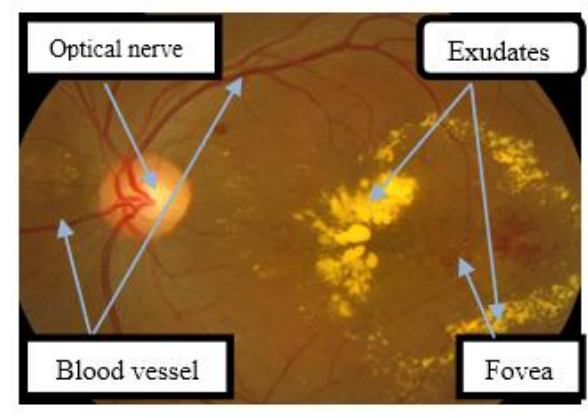

Figure 1.Digital fundus image of human retinal along with its main components and exudates

\section{Background}

The previous research works to identify exudates on retinal fundus images are based on two main criteria i.e., lesion based and image based, for assessing the diagnostic accuracy of exudates detection technique. In lesion-based criterion each abnormal retinal image can be segmented into a number of exudates regions. By considering a set of retinal image and applying an appropriate segmentation technique. The lesion based accuracy can be measured in terms of lesion sensitivity and specificity. The lesion-based accuracy can be assessed either in pixel-level basis or alternatively using large collection of pixel e.g., 10x10 slice. Exudates detection and identification was investigated by Phillips et al. $[15,16]$. The contrast of the exudates are enhanced, then after global and local thresholding values were used to segment exudates lesions. The sensitivity of this technique was reported between $61 \%$ and $100 \%$, based on 14 images.

Ege et al. [17] locates exudates and cotton wool acne in 38 color images. These abnormalities were initially detected using a combination of templates matching, region growing, and thresholding technique. 
Santosh Kumar Mishra, Deepti Mittal and Ramesh Kumar Sunkaria; Designing of Computer Aided Diagnostic System for the

Identification of Exudates in Retinal Fundus Images. Journal of Biomedical Engineering and Medical Imaging, Volume 2, No 3, June (2015) , pp 29-40

Bayesian classifier based technique was classified the bright region into cotton wool spots, exudates and noise. The classification performance was only $62 \%$ for exudates and $52 \%$ for cotton wool spots.

Wang et al. [18] using the minimum-distance discriminate classifier to identify the retinal bright lesion such cotton wool and exudates. Neural network (NN) have been also classify the retinal exudates Another NN based exudates detection work was conducted by Hunter et al. The NN was trained to discriminate exudates from drusen based on $16 \times 16$ pixel slice. This technique achieved $100 \%$ sensitivity and $70 \%$ specificity.

Walter et al. [12] identified exudates from green channel of retinal image. After initial localization, the exudates are determined by mathematical morphology technique. This method had three parameter: size of local window, and two other threshold value. The first threshold find the minimum variation within each local window. The candidate exudates region based on first thresholding value. The second threshold changes the surrounding background pixels to be classify the exudates. This technique was achieved $92.8 \%$ sensitivity and $92.8 \%$. predictivity asset of 15 abnormal retinal images.

Niemeijer et al. [19] distinguished the bright lesion, i.e., exudates, cotton wool spot, and drusen from color retinal images. In the first step, the image pixels are classified, in a lesion probability map that grouped into probable lesion pixel cluster characteristics, each probable cluster was assigned the likelihood that the cluster was a true bright lesion. these clusters were classified as exudate, cotton, wool spot, or drusen.

Goldbaum et al. [20] have discriminated similarly colored objects in retinal images based on color information, not many work have shown interest in color classification of retinal images.

Alireza et al. [21] proposed a method to automatic segment of exudates based on computational intelligent techniques. The color retinal image are segmented using fuzzy c-means clustering following some preprocessing steps, set of initial feature such as color, size, edge strength, and texture are extracted to classify these segmented image initial exudates and non-exudates region.

Ahmed et al. [22] proposed a method to automatically segment optic nerve and exudates. In the algorithms, they used preprocessing steps such as averaging filter, contrast adjustment, Thresholding, morphological opening, watershed transformation on the green component of the image. This method yielded sensitivity $96.7 \%$.

Chugh et al. [23] detection of exudates such as uses homogeneity of healthy areas rather than unhealthy areas. In this method first extracted the healthy area such as optical nerve using sobel filter method and blood vessel by entropy thresholding method. Thresholding method is segmented exudates from diabetic retinopathy images. The proposed method yields accuracy $90 \%$.

\section{Material and method}

\subsection{Materials}

MESSIDOR, a publicly available dataset is used to test performance of segmentation and classification for exudation. The MESSIDOR[24] dataset containing 80 fundus images with exudates region with Zeiss Visucam PRO fundus camera, at resolution of $1449 \times 2201$ pixel and with a $45^{\circ}$ Field of view. The image 
capturing process is vetted by automatic quality assessment algorithms based on the Elliptical local vasculature density feature [25-26].

\subsection{Method}

A method for the identification of exudates in fundus retinal images is presented which will further help in building a computer aided diagnostic system for macular edema. exudate, also known as bright lesions, appears as bright spots and patches in fundus image with highest contrast in the green channel of the color image. An automatic system for detection of exudates should enhance the contrast of bright region with smoothing the dark regions [27]. In this work, morphological reconstruction is used to smooth dark region such as haemorrhages and blood vessels [28]. The objective of contrast enhancement is to improve the contrast of lesion for easy detection. The image normalization technique used for detection all possible bright regions [29]. They are appropriate for texture representation and discrimination. The binary candidate region for exudates was extracted by applying a low adaptive Thresholding value [30]. The region was segmented by the thresholding, enhanced image also contain optical nerve region and pixel due to their similarity with exudates. For correct detection the false and unauthentic pixel should removed before the classification stage. The proposed system segmented optical nerve using masking of related area where optical nerve probability in fundus image data base system.

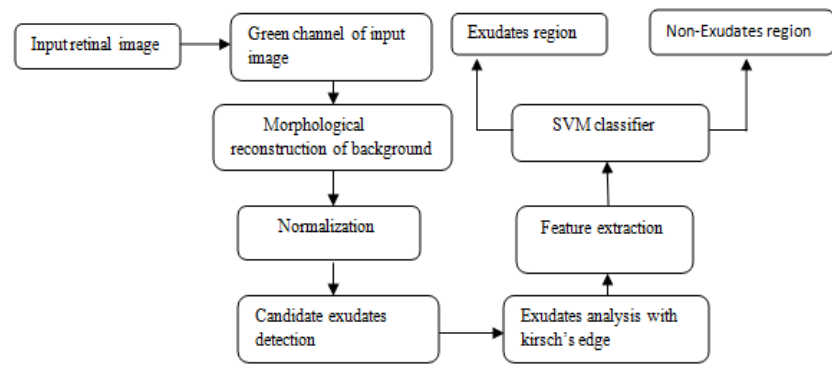

Figure.2. Proposed computer added diagnosis system for the identification of exudates in retinal fundus image

i) Selection: The green channel is selected because of better contrast of this channel then the rest two channels and it will further help in extracting brightest region from background.

ii) Preprocessing: Selected green channel of retinal fundus images are passed through the various preprocessing steps in order to detect the region of exudates. The first step is the application of a big size median filter shown in the work of Niemeijer et.al [31], showed that the size of the median filter should be $1 / 30$ th of the height of the fundus image for background estimation. After that estimated back ground is subtracted from original image. The step has great computational performance advantage by avoiding the multiple passes.

iii) Image normalization: Image normalization is the process where highest intensity of image is centered at zero. We enhanced the normalization with addition of morphological reconstruction [29]. It improved the nerve fiber layer and other structure of edges of optical nerve, without any expansion of exudates region. The histogram showed the clear division between dark structure and bright structure. The dark structure like macula, vasculalature are located at the left side of the histogram. On other hand bright structure are formed on the positive side of the histogram. Which contain the optical nerve, bright lesion 
Santosh Kumar Mishra, Deepti Mittal and Ramesh Kumar Sunkaria; Designing of Computer Aided Diagnostic System for the Identification of Exudates in Retinal Fundus Images. Journal of Biomedical Engineering and Medical Imaging, Volume 2, No 3, June (2015) , pp 29-40

such as exudates and other structure related to very bright retinal pigment epithelium layer. Because of the alignment of the histogram after the normalization we can select all exudates candidate region.

The method of optical nerve removal [32], it is understandable that the similarity of potential of color between optical nerve and exudates in fundus image. The optical nerve detection is the mature technique. So we only concentrate to exudate detection. We did not implement automatic optical nerve detection in our paper. We did manual removal of optical nerve by the having size slightly greater than optical nerve.

iv) Exudate edge detection: The exudate detection is performing by conveying a score for each exudates candidate. The exudate candidates are selected by running 8-neighbour connected component analysis of exudates candidate region. The way of implementation is based on Kirsch's Edges [33]. The advantage of this method to take the higher inner and outer edge value of exudates in compare to non-exudates structure. Kirsch's edges try to capture the external edges of the lesion candidate. This edge detection is based on kernel $\mathrm{K}$ to evaluate at 8 different directions. The kernel output are combined together by selecting the maximum value found on each pixel output

It computes the gradient by convolution the image with eight template impulse response arrays as shown in figure. The scalar factor is $1 / 15$.

$$
\mathrm{K}=\begin{array}{ccc}
\frac{5}{15} & \frac{-3}{15} & \frac{-3}{15} \\
\frac{5}{15} & \mathrm{O} & \frac{-3}{15} \\
\frac{5}{15} & \frac{-3}{15} & \frac{-3}{15}
\end{array}
$$

The gradient of different direction is obtained by convolving the image with eight impulse response arrays. The final gradient is set to be a large gradient among different direction. Thus edge is enhanced by Kirsch's method. The threshold is set after edge enhancement to determine if a pixel belongs to the edge or not.

Thresholding based technique focus on a global or adaptive gray level analysis, but the automatic selection of proper Thresholding is difficult due to uneven illumination of the image.

v) Feature Extraction: Feature extraction involves simplifying the amount of resources required to describe a large set of data accurately. The large number of variable generally requires a large number of memory and computational power or a classification algorithm. Texture plays a significant role in image analysis and pattern recognition. In this proposed method, Gray level co-occurrence matrix (GLCM) is formulated to obtain statistical texture features. There are two types of texture feature measures. They are first order and second order measures. GLCM is the second order texture calculation. In second order texture measures consider relationship between neighbors. But first order texture measures are statics, consider not pixel neighbor relation. Texture features have high discrimination accuracy, requires less computation time and hence efficiently used for real time Pattern recognition applications.

GLCM is defined over an image to be distribution of co-occurrence values at a given offset; GLCM is a matrix where the number of row and columns is equal to the number of gray level. The use of statistical feature is one of the early proposed methods in the image processing literature. Haralic [34] suggested the use of co-occurrence matrix of GLCM. The feature extraction from GLCM is Energy, Contrast, Entropy, Correlation and Homogeneity.

1. Contrast- it is a measurement of intensity contrast between a pixel and its neighborhood pixels in the whole image. 


$$
\text { Contrast }=\sum_{i, j}|i-j|^{2} p(i, j)
$$

2. Correlation - It is a measure of how a pixel is correlated to its neighborhood pixel in whole image.

$$
\text { Correlation }=\sum_{i, j} \frac{\left(\left(i-\mu_{i}\right)\left(j-\mu_{j}\right) p(i-j)\right)}{\sigma_{i} \sigma_{j}}
$$

3. Energy - It is the sum of squared elements in GLCM. It ranges from 0 to 1 . For constant image, energy value is 1.

$$
\text { Energy }=\sum_{i, j} p(i, j)^{2}
$$

4. Homogeneity - The closeness measurement of the elements distribution in GLCM to GLCM diagonal.

$$
\text { Homogeneity }=\sum_{i, j} \frac{p(i, j)}{1+|i, j|}
$$

vi) Classification: Classification based technique supervised approaches attempt to extract different feature and then use a classifier to classify the exudates and non-exudates region. Support vector machine (SVM) classifier is used to classify the Exudates and Non-Exudates regions. SVM is a supervised learning model with associated learning algorithms that analyze data and recognize patterns, used for classification and regression analysis. The basic SVM takes a set of input data and predicts, for each given input. The classification process is divided into training phase and testing phase. The known data is the training phase and unknown data is the given in testing phase. The accuracy depends on the efficiency of the classification.

vii) Measurement and performance evaluation: Different measures are used to evaluate the performance of the system. The measures used are Accuracy, Mathews correlation coefficient (MCC), Sensitivity, Specificity and Positive prediction value (PPV). These values are calculated from the Confusion Matrix [35]. A confusion matrix contains information about actual and predicted classifications done by a classification system. Performance of such systems is commonly evaluated using the data in the matrix.

TN (True negative) - correct prediction as normal

FN (False negative) - incorrect prediction of normal

FP (False positive) - incorrect prediction of abnormal

TP (True positive) -correct prediction of abnormal

The accuracy is the percentage of prediction that is correct

$$
\text { Accuracy }=(T P+T N) \div(T P+F P+T N+F N)
$$


Santosh Kumar Mishra, Deepti Mittal and Ramesh Kumar Sunkaria; Designing of Computer Aided Diagnostic System for the Identification of Exudates in Retinal Fundus Images. Journal of Biomedical Engineering and Medical Imaging, Volume 2, No 3, June (2015) , pp 29-40

The Matthews correlation coefficient is used in machine learning as a measure of the quality of binary classifications.

$$
\mathrm{MCC}=(T P \times T N-F P \times F N) \div(\sqrt{(T P+F P)(T P+F N)(T N+F P)(T N+F N)})
$$

The sensitivity is the percentage of positive level instances that were predicted as positive.

$$
\text { Sensitivity }=(T P) \div(T P+F N)
$$

The specificity is the percentage of negative labeled instance that were predicted as negative.

$$
\text { Specificity }=(T N) \div(T N+F P)
$$

The Positive Prediction Value or precision rate is the percentage of positive prediction is correct.

$$
\mathrm{PPV}=(T P) \div(T P+F P)
$$

\section{Result and discussion}

\subsection{Green channel separation:}

The abnormalities are more visible in the green channel. So separation of green channel from RGB images is needed. The separate green channel images is show in figure 3(a).

\subsection{Median Filter and Background estimation}

\subsubsection{Median filter:}

In the green channel, noise is present. Hence median filter is using. The median filter is non linear digital filter technique. It's proved to be best in removing salt \& pepper and Impulse noise. Median filter erased black dots called the pepper and fills in white holes in the image, called salt. It better works than mean filter by preserving sharp edges. It simply replaces each pixel value by the median of the intensity level in the neighborhood of the pixel. As can be seen figure 3(b). Here using the big size window of median filter because saving the bright region of the image.

\subsubsection{Background estimation:}

Due to using median filter some bright region information are lost. For recovering of that information we are estimating the background. As shown in figure 3(c). In retinal image database containing $1449 \times 2201$ pixel configurations, where 1449 rows are present there. So in result (49x49) size of median filter window, this result is good contrast between exudates and background. This approach has great computational performance advantage avoiding the multiple passes. After that estimated background is subtracted from the original image in order to obtain a normalized version, in proposed method, enhanced the normalization with morphological reconstruction.

\subsection{Morphological reconstruction:}

In morphological reconstruction process one image called the marker, based on the character of another image, called masked. The high point and the peak in the marker image specify where processing begins. The peaks spared out or dilate while being forced to fit within the mask image. The scattering processing continues until the image value stop changing as shown in figure 3(e). 


\subsection{Image normalization:}

In image normalization technique, the dark and bright region is classified shown in figure 3(e). Histogram of normalized image shown that all pixels centered at zero shown in figure $3(f)$. The normalized image is called exudate candidate detection.

\subsection{Edge detection and global thresholding:}

After image normalization we want to detect the external edge of the exudates candidate region, Using Kirsch's edge detection. Kirsch's operator is non-linear edge detector that find maximum edge strength in few predetermine direction. The operator takes a single kernel mask and rotates it in $45^{\circ}$ increment through all compass direction. The edge magnitude of the kirsch operator is calculated as the maximum magnitude across all direction, and the resultant image is shown in figure $2(\mathrm{f})$.

Global thresholding is used to convert an intensity image to binary image. Level is a normalized intensity value that lies in the range [01]. Then threshold to minimize the intra class variance of the black and white pixels. Final exudates segmented image is shown in figure $3(\mathrm{~g})$.

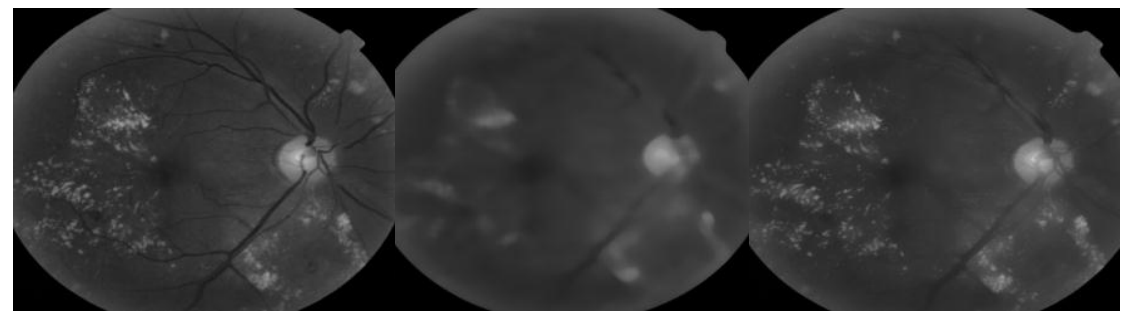

( a)

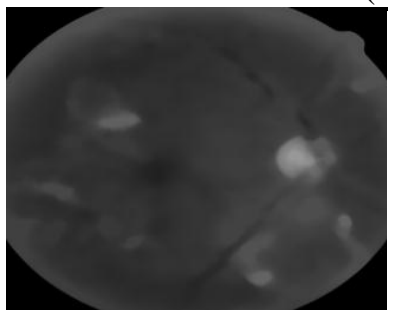

(d) (b)

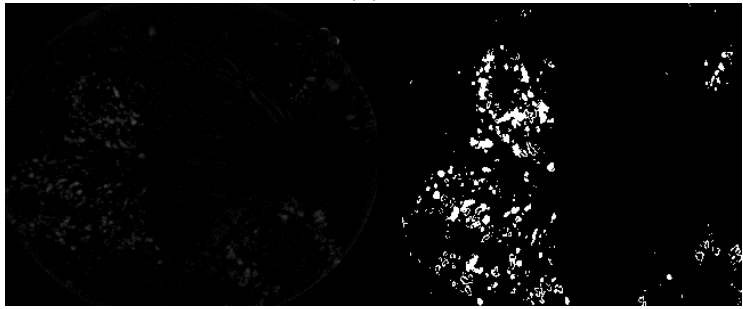

(f) $(\mathrm{g})$

Figure.3. a, b, c, d, e, f, g: green channel image, median filtered image, background estimation image, morphological image, histogram of normalized image, exudates candidate detection, exudates segmented image.

\subsection{Feature extraction:}

The GLCM feature of exudates and non-exudates segmented region of the image is extracted. The size of the exudates and non-exudates region is equal. The segmented region of exudates and non-exudates regional size is $(10 \times 10)$ pixel.

The randomly select the slice of 42 exudates and 40 non-exudates region from 9 different retinal fundus images. And find the mean \pm variance of GLCM properties like contrast, correlation, energy and homogeneity of exudates and non-exudates regions. 
Santosh Kumar Mishra, Deepti Mittal and Ramesh Kumar Sunkaria; Designing of Computer Aided Diagnostic System for the Identification of Exudates in Retinal Fundus Images. Journal of Biomedical Engineering and Medical Imaging, Volume 2, No 3, June (2015) , pp 29-40

Table1: GLCM feature values in terms of mean \pm variance

\begin{tabular}{|c|c|c|c|c|}
\hline Mean \pm Variance & Contrast & Correlation & Energy & Homogeneity \\
\hline Exudates & $0.1041 \pm 0.0032$ & $0.7652 \pm 0.0245$ & $0.4898 \pm 0.0187$ & $0.9591 \pm 0.0008$ \\
\hline Non-exudates & $0.1825 \pm 0.0156$ & $0.3329 \pm 0.0334$ & $0.6031 \pm 0.0524$ & $0.9087 \pm 0.0038$ \\
\hline
\end{tabular}

\subsection{SVM classifier:}

For the proposed work, 82 slices of images were chosen randomly feature is extracted and its classification was obtained. The construct feature sets are separately tested using the SVM classifier. The SVM structure depends on the training set and testing set from feature like area, contrast, correlation, and homogeneity are given as input to SVM. To train the network 82 retinal images were taken than the system is loaded with the statistical features, which are, contrast, correlation, energy, and homogeneity of retinal image from the workspace or from file. There are 17 images of testing for exudates region and corresponding 15 testing image for Non-exudates region .The computational result are presented

Table2: Confusion matrix for exudates classification

\begin{tabular}{|c|c|}
\hline Parameter & Segmented exudates \\
\hline TP & 15 \\
\hline FP & 0 \\
\hline FN & 2 \\
\hline TN & 15 \\
\hline
\end{tabular}

The performance of proposed system is measured using sensitivity, specificity, PPV, accuracy and MCC. Sensitivity is true positive rate. Specificity is true negative rate. Table 3 clearly shows that the designed computer aided system to diagnose AMD out performs the other existing methods in terms of its performance by measuring Sensitivity, specificity, PPV, Accuracy, MCC.

Table 3: Comparative performance evolution of proposed computer aided diagnostic system with existing method

\begin{tabular}{lccccc}
\multicolumn{1}{c}{ Method } & Sensitivity & Specificity & PPV & Accuracy & MCC \\
\hline Wang et al [18] & - & 70 & - & - & - \\
Walter et al [12] & 92.74 & 100 & 92.39 & - & - \\
Ahmed et al [22] & 96.7 & 100 & 94.9 & - & - \\
Osareh et al [21] & 93 & 94.1 & - & 93.4 & - \\
Akram et al [5] & - & - & - & - & 94.73 \\
Proposed method & 88.23 & 100 & 100 & 93.75 & 88.23 \\
\hline
\end{tabular}

\section{Conclusions}

The proposed work presents the designing of computer aided diagnostic system for identification of exudates in retinal fundus images. System consists of three phases these are candidate exudates detection, feature extraction, and classification. The bright region is enhanced and segmented using morphological reconstruction, image normalization and global thresholding. Normalization procedure gives a substantial computational advantage to our method. The median filter and morphological reconstruction gives good contrast of foreground image. Feature set of each candidate region is formed using different properties of exudate and non-exudate region. We implemented a SVM based classifier to 
divide the region into exudate and non-exudate regions. The results demonstrated that the proposed system can be used in computer aided diagnosis system for DR as it identified and detected exudates with high accuracies.

\section{REFERENCES}

[1] D. E. Singer, D. M. Nathan, H. A. Fogel, and A. P. Schachat, "Screening for diabetic retinopathy." Ann Intern Med, vol. 116, no. 8, 1992. P: 660-671.

[2] M. D. Abramoff, M. Niemeijer, M. S. A. Suttorp-Schulten, M. A. Viergever, S. R. Russell, and B. van Ginneken, "Evaluation of a system for automatic detection of diabetic retinopathy from color fundus photographs in a large population of patients with diabetes." Diabetes Care, vol. 31, no. 2, 2008. P: 193-198.

[3] S. Philip, A. D. Fleming, K. A. Goatman, S. Fonseca, P. McNamee, G. S. Scotland, G. J. Prescott, P. F. Sharp, and J. A. Olson, "The efficacy of automated "disease/no disease" grading for diabetic retinopathy in a systematic screening programme." $\mathrm{Br} J$ Ophthalmol, vol. 91, no. 11, 2007.P:1512-1517.

[4] M.D. Abramoff, M.K. Garvin, Sonka, M., "Retinal Imaging and Image Analysis," IEEE reviews in Biomedical Engineering, vol.3, 2010. P:169.

[5] M. U. Akram and S. A. Khan, "Automated detection of dark and bright lesions in retinal images for early detection of diabetic retinopathy", Journal of Medical System, vol.36, no.5, 2011.P:3151-3162.

[6] A. Tariq, M. U. Akram, A. Shaukat, S. A. Khan, "Automated Detection and Grading of Diabetic Maculopathy in Digital Retinal Images", Journal of Digital Imaging, vol. 26, no. 4, 2013. P: 803812, 2013.

[7] M. U. Akram, A. Tariq, M. A. Anjum, M. Y. Javed, "Automated Detection of Exudates in Colored Retinal Images for Diagnosis of Diabetic Retinopathy", OSA Journal of Applied Optics, vol. 51 no. 20,2012.P: 4858-4866.

[8] R. Phillips, J. Forrester, and P. Sharp, "Automated detection and quantification of retinal exudates," Graefes Arch Clin Exp Ophthalmol, vol. 231, no. 2,1993. P: 90-94.

[9] C. Sinthanayothin, J. F. Boyce, T. H. Williamson, H. L. Cook, E. Mensah, S. Lal, and D. Usher, "Automated detection of diabetic retinopathy on digital fundus images," Diabetic Medicine, vol. 19, no. 2,2002.P: 105-112.

[10] H. Li and O. Chutatape, "Automated feature extraction in color retinal images by a model based approach," IEEE Transactions on Biomedical Engineering, vol. 51, no. 2, 2004. P: 246-254. 
Santosh Kumar Mishra, Deepti Mittal and Ramesh Kumar Sunkaria; Designing of Computer Aided Diagnostic System for the Identification of Exudates in Retinal Fundus Images. Journal of Biomedical Engineering and Medical Imaging, Volume 2, No 3, June (2015) , pp 29-40

[11] T. Walter, J. K. Klein, P. Massin, and A. Erginay, "A contribution of image processing to the diagnosis of diabetic retinopathy-detection of exudates in color fundus images of the human retina." IEEE Transactions on Medical Imaging, vol. 21, no. 10, 2002.P: 1236-1243.

[12] A. Sopharak, B. Uyyanonvara, S. Barman, and T. H. Williamson, "Automatic detection of diabetic retinopathy exudates from non-dilated retinal images using mathematical morphology methods." Computerized Medical Imaging and Graphics, vol. 32, no. 8, 2008.P: 720-727.

[13] D. Mittal, V. kumar, S. C. Saxena, N.Khandelwal, "Neural networks based focal liver diagnosis using ultrasound images,"Computerized Medical Imaging and Graphics, vol.35, no-4,2011.P:135323.

[14] K. Kumari, D. Mittal, "Automated Drusen Detection Technique for Age-Related Macular Degeneration" Society for science and education, united kingdom,vol.2,2015,P:19-26.

[15] R. Phillips, T. Spencer, P. Ross, P. Sharp, and J. Forrester, "Quantification of diabetic maculopathy by digital imaging of the fundus," Eye, vol. 5, 1991.P: 130-137.

[16] R. Phillips, J. Forrester, and P. Sharp, "Automated detection and quantification of retinal exudates," Graefe's Arch. Clin. Exp. Ophthalmol., vol. 231, 1993.P: 90-94, 1993.

[17] B. Ege, O. Larsen, and O. Hejlesen, "Detection of abnormalities in retinal images using digital image analysis," in Proc. 11th Scand. Conf. Image Process., vol. 13,2009.P: 833-840.

[18] H. Wang, H. Hsu, K. Goh, and M. Lee, "An effective approach to detect lesions in retinal images," in Proc. IEEE Conf. Comput. Vis. Pattern Recogn., Hilton Head Island, vol. 2, 2000. P: 181-187.

[19] M. Niemeijer, B. V. Ginneken, S. R. Russell, M. Suttorp, and M. D. Abramoff, "Automated detection and differentiation of drusen, exudates and cotton-wool spots in digital color fundus photographs for diabetic retinopathy diagnosis," Invest. Ophthalmol. Vis. Sci., vol. 48, 2007. P: 2260-2267.

[20] M. Goldbaum, S. Moezzi, A. Taylor, and S. Chatterjee, "Automated diagnosis and image understanding with object extraction, object classification and inferencing in retinal images," in Proc. IEEE Int. Conf. Image Process., Lausanne, Switzerland, vol. 3,1996.P: 695-698.

[21] Alireza O., B. Shadgar and R. Markham, "A Computational-Intelligence- Based Approach for Detection of Exudates in Diabetic Retinopathy Images", IEEE Trans on Information Tech in Biomedicine, vol. 13, No.4, 2009. P: 535-545.

[22] Ahmed Wasif Reza, C. Eswaran, Subhas Hati, "Automatic Tracing of Optic Disc and Exudates from Color Fundus Images Using Fixed and Variable Thresholds", Journal of Medical Systems, vol. 33,2009.P: 7380. 
[23] S. Chugh, J Kaur, and D. mittal,"Exudates segmentation in retinal fundus image for detection of Diabetic retinopathy", IJERT,vol.3,2014. P:673-677.

[24] Methods to evaluate segmentation and indexing techniques in the field of retinal ophtalmology. [Online]. Available: http://messidor.crihan.fr

[25] L. Giancardo, M. Abramoff, E. Chaum, T. Karnowski, F. Meriaudeau, and K. Tobin, "Elliptical local vessel density: a fast and robust quality metric for retinal images," in Conf. of the IEEE EMBS, 2008.

[26] L. Giancardo," Quality Assessment of Retinal Fundus Images using ELVD". IN-TECH, 2010, ch. New Developments in Biomedical Engineering, 2010.P: 201-224.

[27] D. Mittal, V.Kumar, S.C.saxena, N. khandewal and N. kalra" Enhancement of the ultrasound images by modified anisotropic diffusion method".Med. Biol.Engg. computer., vol.48, no12,2010.P:1281-1291

[28] L. Vincent, "Morphological grayscale reconstruction in image analysis: applications and efficient algorithms," IEEE Journal of Image Processing, vol. 2, no. 2, 1993.P: 176-201.

[29] A. D. Fleming, S. Philip, K. A. Goatman, J. A. Olson, and P. F. Sharp,“Automated microaneurysm detection using local contrast normalization and local vessel detection," IEEE Transactions on Medical Imaging, vol. 25, no. 9,2006.P: 1223-1232..

[30] C. I. Sanchez, M. Garca, A. Mayo, M. I. Lopez, and R. Hornero, "Retinal image analysis based on mixture models to detect hard exudates." Medical Image Analysis, vol. 13, no. 4, 2009. P: 650658.

[31] M. Niemeijer, B. van Ginneken, J. Staal, M. S. A. Suttorp-Schulten, and M. D. Abramoff, "Automatic detection of red lesions in digital color fundus photographs," IEEE Trans Med Imag, vol. 24, no. 5, 2005.P: 584- 592.

[32] J. Kaur, D. Mittal " Segmentation and Measurement of Exudates in Fundus Images of the Retina for Detection of Retinal Disease."Society for science and education, united kingdom, vol.2.2015.P:28-38.

[33] R. A. Kirsch, "Computer determination of the constituent structure of biological images." Computers and Biomedical Research, vol. 4, no. 3,1971.P: 315-328.

[34] K. Shanmugam, R. M. Haralick and I. H. Dinstein, "Textural features for image classification" IEEE Transactions on Systems, Man and Cybernetics 3, 1973.P: $610-621$.

[35] R. Kohavi and F. Provost. Glossary of terms, Special Issue on "Applications, of Machine Learning and the Knowledge Discovery Process", Journal of Machine Learning, vol. 30, 1998. P: 271-274. 